\title{
Anomaly Detection for Resilient Control Systems Using Fuzzy- Neural Data Fusion Engine
}

\section{International Symposium on Resilient Control Systems}

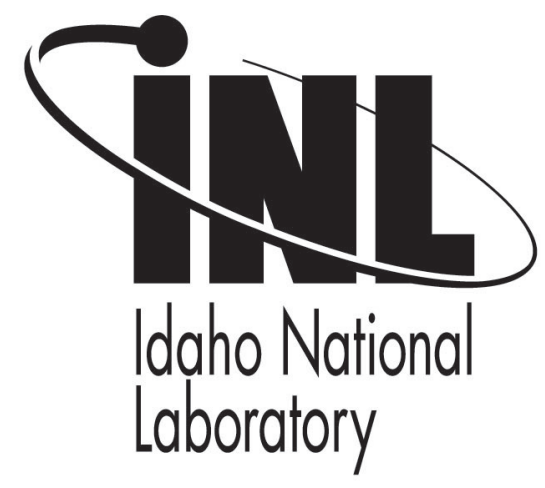

\section{Ondrej Linda Milos Manic \\ Timothy R. McJunkin}

\section{August 2011}

This is a preprint of a paper intended for publication in a journal or proceedings. Since changes may be made before publication, this preprint should not be cited or reproduced without permission of the author. This document was prepared as an account of work sponsored by an agency of the United States Government. Neither the United States Government nor any agency thereof, or any of their employees, makes any warranty, expressed or implied, or assumes any legal liability or responsibility for any third party's use, or the results of such use, of any information, apparatus, product or process disclosed in this report, or represents that its use by such third party would not infringe privately owned rights. The views expressed in this paper are not necessarily those of the United States Government or the sponsoring agency. 


\section{Anomaly Detection for Resilient Control Systems Using Fuzzy-Neural Data Fusion Engine}

\author{
Ondrej Linda, Milos Manic \\ University of Idaho \\ Idaho Falls, ID, USA \\ olinda@uidaho.edu,misko@ieee.org
}

\author{
Timothy R. McJunkin \\ Idaho National Laboratory \\ Idaho Falls, ID, USA \\ Timothy.McJunkin@inl.gov
}

\begin{abstract}
Resilient control systems in critical infrastructures require increased cyber-security and state-awareness. One of the necessary conditions for achieving the desired high level of resiliency is timely reporting and understanding of the status and behavioral trends of the control system. This paper describes the design and development of a fuzzy-neural data fusion system for increased state-awareness of resilient control systems. The proposed system consists of a dedicated data fusion engine for each component of the control system. Each data fusion engine implements three-layered alarm system consisting of: 1) conventional threshold-based alarms, 2) anomalous behavior detector using self-organizing maps, and 3) prediction error based alarms using neural network based signal forecasting. The proposed system was integrated with a model of the Idaho National Laboratory Hytest facility, which is a testing facility for hybrid energy systems. Experimental results demonstrate that the implemented data fusion system provides timely plant performance monitoring and cyber-state reporting.
\end{abstract}

Index Terms - Artificial Neural Network, Self-Organizing Map, Fuzzy Logic System, Data-Fusion, Control System, Resiliency, Hytest.

\section{INTRODUCTION}

$\mathrm{R}$ ESILIENT control systems demand implementation of robust cyber-security environment together with ensuring increased state-awareness. This goal can be achieved via complex system monitoring, real-time system behavior analysis and timely reporting of the system state to the responsible human operators [1].

This paper describes the development and implementation of fuzzy-neural based data fusion system. The main purpose of the data fusion system is to provide real-time monitoring and analysis of complex critical control systems. The data fusion system evaluates the state of the plant by calculating anomaly indicators associated with different components of the system. These anomaly indicators are then delivered to the operator via an HMI.

The proposed data fusion architecture uses computational intelligence algorithms such as artificial neural networks and fuzzy logic for system modeling, signal forecasting and intelligent control. Several other approaches for plant monitoring can be found in literature. Fuzzy logic has been previously used for monitoring sensory data and alarm processing in nuclear power plants in [2]-[6]. Neural networks have been applied to nuclear reactor monitoring in [7], [8]. The concept of control charts was applied towards designing a pre-alarm system in nuclear power plant control rooms in [9]. In [10], the fusion of support vector machines and adaptive neuro-fuzzy inference system was used for fault detection and diagnosis in industrial steam turbines. The cyber-security of critical infrastructure control systems was also analyzed using fuzzy logic and artificial neural networks in [11], [12]. However, majority of the previously published work was applied to rather small system and the cyber-security element was rarely considered. The presented work develops a data fusion engine tailored to the specifics of complex resilient control systems such as the Idaho National Laboratory (INL) Hytest facility for testing hybrid energy systems [13], [14].

The developed data fusion system uses two different artificial neural networks architectures to model the normal behavior of the plant and its control system. Firstly, a SelfOrganizing Map (SOM) is used to cluster the available normal behavior data. During the testing phase, individual neurons of the SOM are transformed into fuzzy rules. These fuzzy rules calculate the degree of similarity of the observed behavior with the previously known normal behavior patterns. Secondly, feed-forward neural network is used to forecast the future measurements for each sensor based on the previously observed history. The predicted measurements are then matched to the true observation and the prediction error is fused into a robust anomaly indicator using a fuzzy logic controller. The proposed data fusion system was integrated with a Matlab Simulink model of the INL Hytest facility.

The rest of the paper is organized as follows. Section II provides fundamental overview of the two considered neural network architectures, the feed-forward neural network and the self-organizing maps. Sections III presents the developed neural-network based data-fusion system. Section IV describes the experimental Hytest system and shows the test results. The paper is concluded in Section V. 


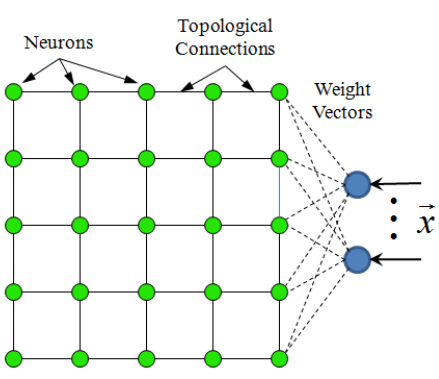

(a)

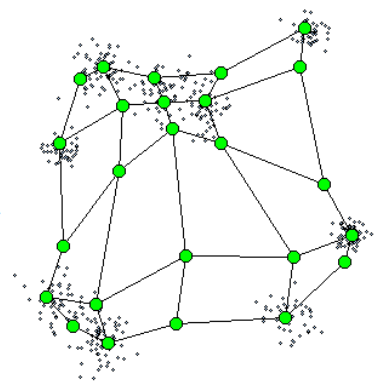

(b)
Fig. 1 Self-Organizing Map displayed in the output space (a) and in the input space adapted to 2D distribution of input points (b).

\section{ARTIFICIAL NEURAL NETWORKS}

The implemented data fusion system utilizes two diverse paradigms of artificial neural-networks - the supervised feedforward Neural Networks (NN) and the unsupervised SelfOrganizing Maps (SOM). This section provides some of the fundamentals of both neural network architectures.

\section{A. Feed-Forward Neural Networks}

A feed-forward $\mathrm{NN}$ is composed of multiple interconnected layers, each consisting of several neurons. The training of the NN proceeds in a supervised manner. The gradient descent approach is used to optimize system parameters based on the error of the network's output. Here, a specific combination of the Error Back-Propagation and the Levenberg-Marquardt EBP-LM algorithm was used [15]-[17].

First an input vector $\vec{x}=\left\{x_{1}, \ldots, x_{m}\right\}$ is provided to the input layer of NN. The net input value of neuron $i$ in layer $k+1$ is then calculated as the weighted sum of the input connections:

$$
n^{k+1}(i)=\sum_{j=1}^{S k} w^{k+1}(i, j) a^{k}(j)+b^{k+1}(i)
$$

Here $S k$ denotes the number of neurons in layer $k, w^{k+1}(i, j)$ is the weight of the connection from neuron $j$ in layer $k$, $b^{k+1}(i)$ is the bias of neuron $i$ and $a^{k}(j)$ is the output from neuron $j$ in layer $k$.

The output of neuron $i$ in layer $k+1$ is:

$$
a^{k+1}(i)=f^{k+1}\left(n^{k+1}(i)\right)
$$

Here $f^{k+1}$ is the activation function of neuron $i$. Typically, a sigmoidal or linear activation function is used.

For a neural network with $L$ layers, the task of the EBP-LM algorithm is to minimize the total error:

$$
E=\sum_{p=1}^{P} \sum_{m=1}^{M}\left(d_{p m}-a_{p m}^{L}\right)^{2}
$$

which can be reduced to:

$$
E=\sum_{p=1}^{P} \sum_{m=1}^{M}\left(e_{p m}\right)^{2}
$$

Here $P$ and $M$ are the number of patterns and the number of outputs respectively, and $d_{p m}$ denotes the desired output.

The weight update rule for the EBP-LM algorithm is derived from the Newton's method using the Hessian and the gradient of system parameters. For the error function $E$, which is a sum of squares, the Hessian and the gradient can be computed as the Jacobian of the partial derivative of error with respect to the weights. The Jacobian matrix is computed by a modified EBP algorithm [17].

\section{B. Self-Organizing Maps}

The Self-Organizing Map (SOM) algorithm was developed in 1981 [18]. SOM uses unsupervised winner-takes-all competitive learning method together with cooperative adaptation to adjust its parameters to the topology of the input dataset. The SOM consists of a topological grid of neurons typically arranged in 1D or 2D lattice [19]. The fixed grid defines the spatial neighborhood of each neuron.

Each neuron maintains a synaptic weight vector $\vec{w}=\left\{w_{1}, \ldots, w_{m}\right\}$, where $m$ is the dimensionality of the input space. The input dataset consists of input patterns that can be denoted as $\vec{x}=\left\{x_{1}, \ldots, x_{m}\right\}$. The structure of a 2D SOM is depicted in Fig. 1(a). All neurons are first randomly initialized and then iteratively adapted based on the training set of input data. The training process can be described in several steps as follows [19]:

Step 1 - Initialization: Randomly initialize all synaptic weight vectors in the input domain.

Step 2 - Selection: Select a random input pattern $\vec{x}$.

Step 3 - Competitive Learning: Find the Best Matching Unit (BMU) for the current input pattern $\vec{x}$. The BMU is found by minimizing the Euclidean distance between the input pattern $\vec{x}$ and the synaptic weight vectors $\vec{w}$ :

$$
B M U(\vec{x})=\underset{j}{\operatorname{argmin}}\left\|\vec{x}-\vec{w}_{j}\right\|, j=1,2, \ldots, N
$$

Here, $B M U(\vec{x})$ is the BMU for input pattern $\vec{x}$, operator || $\|$ denotes the Euclidian distance, and $N$ is the number of neurons in the SOM.

Step 4 - Cooperative Updating: Update the synaptic weight vectors of all neurons in SOM using the cooperative update rule:

$$
\vec{w}_{j}(n+1)=\vec{w}_{j}(n)+\eta(n) h_{j, B M U(\vec{x})}(n)\left(\vec{x}-\vec{w}_{j}(n)\right)
$$

Here, $n$ denotes the iteration, $\eta(n)$ is the learning rate and $h_{j, B M(\vec{x})}(n)$ is the value of the neighborhood function for the neuron $j$ centered at $B M U(\vec{x})$.

Step 5 - Convergence Test: Until a specified convergence criterion is met go to Step 2 (e.g. based on the average weight update).

The learning process is controlled by the dynamic learning rate and neighborhood function. The neighborhood function is typically implemented as a Gaussian function centered at the 
selected wining neuron. In order to enforce a convergent behavior the size of neighborhood is reduced by decreasing the standard deviation of the Gaussian function. The learning rate $\eta$ controls the rate of adaptation of individual neurons. Its value is also typically exponentially decayed with the elapsed training time. An illustrative example of a 2D SOM in the input space adapted to a $2 \mathrm{D}$ distribution of data is shown in Fig. 1(b)

\section{NeURAL-NETwork Based Data-Fusion System}

This Section describes the proposed architecture of the fuzzy-neural data fusion system for improved resiliency of critical infrastructures. First, an architecture overview is presented. Next, individual blocks of the data fusion system are described in more detail.

\section{A. Architecture Overview}

The proposed data fusion system architecture is depicted in Fig.2. The system consists of three main blocks: Human Machine Interface (HMI), on-line processing, and the knowledge base. The knowledge base block is constructed offline based on the acquired training data. The information stored in the knowledge base is used to drive the on-line anomaly detection. The on-line processing block monitors and analyzes the incoming data stream of sensory measurements. The incoming measurements are passed through a sequential three-layered anomaly detection system, which evaluates the potential presence of an anomaly. Finally, the generated alarms are forwarded to the HMI and presented to the operator.

The first layer of the on-line processing block consists of conventional min-max bounds on the measurements for normal operating conditions. When the incoming measurements reach outside the normal behavior interval, an alarm is immediately reported. The second layer consists of SOM, which was trained to model the previously observed normal behavior patterns. The on-line processing engine interprets each neuron of the SOM as a fuzzy rule, which is then applied to the incoming signal. The set of SOM based fuzzy rules determines the similarity of the current plant behavior with the previously observed patterns. The third layer consists of feed-forward NN behavior forecaster, which processes the previous history of plant behavior and predicts the near future patterns. These predicted patterns are then compared to the future real plant behavior and significant deviations are reported as prediction error. The prediction error from multiple sensors is fused into a robust anomaly indicator by a fuzzy logic controller.

\section{B. SOM based Anomaly Detection}

The conventional min-max bounds restrict the admissible system behavior into a multi-dimensional hyper-cube. Unfortunately, as long as the anomalous plant behavior stays within this min-max hyper-cube, the intrusion/anomaly will remain undetected. In order to alleviate this issue, the previously observed normal behavior is modeled using SelfOrganizing Map. This SOM trained on the approved normal behavior constitutes a normal behavior model stored in the knowledge base of the data-fusion system.

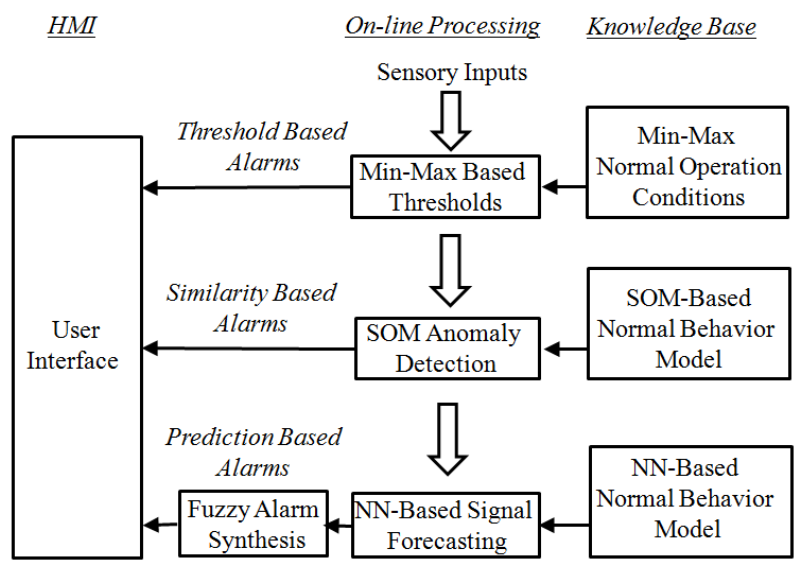

Fig. 2 The fuzzy-neural data fusion system.

The implemented SOM is 2D lattice of $C$ neurons. The input vector $X_{t}$ is constructed from the previous and the current sensory measurements as follows:

$$
X_{t}=\left\{\vec{x}_{t}, \vec{x}_{t-1}, \vec{x}_{t}-\vec{x}_{t-1}\right\}
$$

The SOM structure is trained on the approved normal behavior data until a convergence criterion is met. The trained SOM maps the topology of the normal data within the minmax bounds hyper-cube.

During the on-line processing stage, each neuron of the SOM is transformed into a fuzzy rule. Each fuzzy rule describes the similarity of particular input vector with a specific region of the min-max bound hyper-cube. The fuzzy rules use Gaussian membership functions, which can be described using two parameters mean $m_{j}$ and spread $\delta_{j}$. Parameter $m_{j}$ is set equivalent to the weight vector of neuron $j$. The spread parameter $\delta_{j}$ is set according to the average distance to the immediate topological neighbors of neuron $j$ in the 2D SOM lattice.

The strength of firing of specific rule $R_{j}$ can be computed using the minimum operator as follows:

$$
\mu_{R_{j}}\left(X_{t}\right)=\min _{i=1 . . . n}\left\{\mu_{R_{j}^{i}}\left(X_{t}^{i}\right)\right\}
$$

Here, index $i$ denotes the $i^{\text {th }}$ component of the input space. In this application, the output of each fuzzy rule is a singleton fuzzy set expressing the belonging of input pattern $X_{t}$ to the

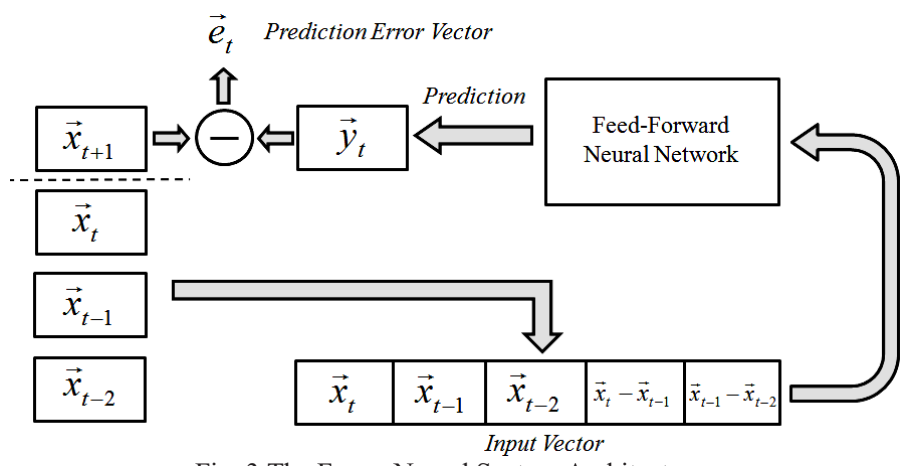

Fig. 3 The Fuzzy-Neural System Architecture. 


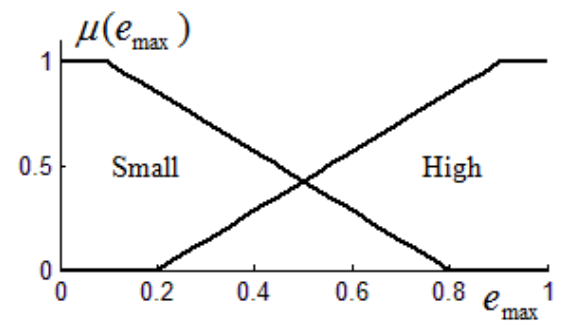

(a)

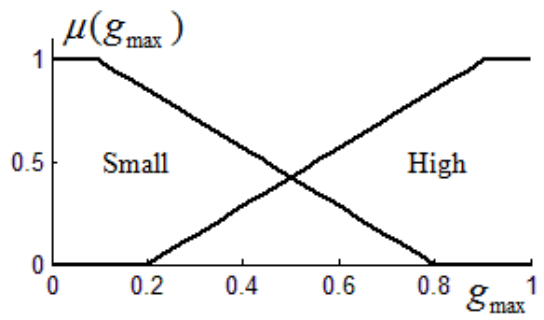

(b)

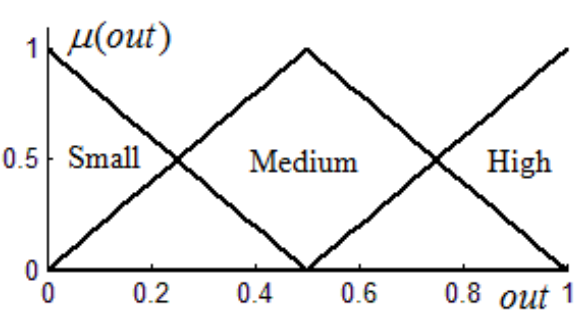

(c)

Fig. 4 Input fuzzy sets (a), (b) and output fuzzy sets (c) for the prediction error based anomaly indicator FLC

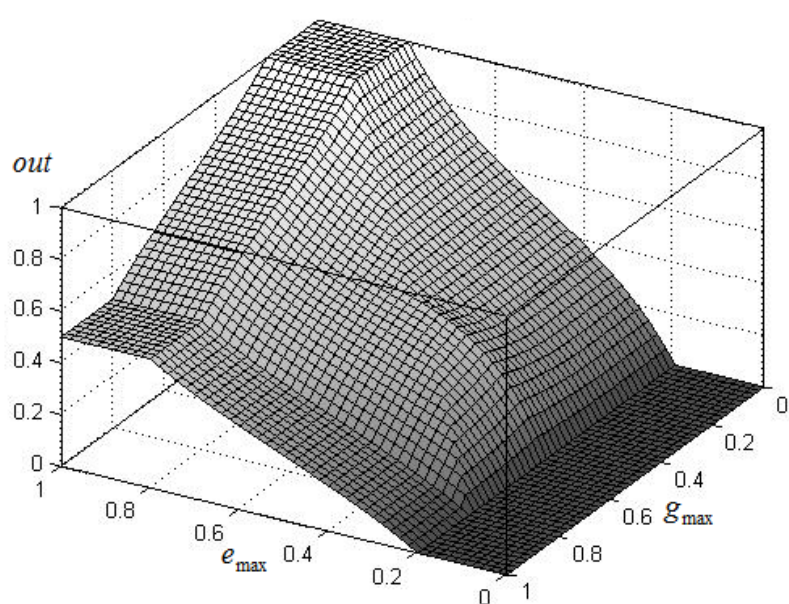

Fig. 5 Fuzzy logic control surface of the anomaly indicator.

TABLE I

FUZZY RULE TABLE

\begin{tabular}{c|c|c}
\multicolumn{3}{|c}{ FUZZY RULE TABLE } \\
\hline \hline$e_{\max } / g_{\max }$ & Small & High \\
\hline $\begin{array}{c}\text { Small } \\
\text { High }\end{array}$ & $\begin{array}{c}\text { Small } \\
\text { High }\end{array}$ & $\begin{array}{c}\text { Small } \\
\text { Medium }\end{array}$
\end{tabular}

normal behavior class. Hence, the output of a particular fuzzy rule is its own firing strength $\mu_{R_{j}}\left(X_{t}\right)$. The output of all rules is aggregated using the maximum operator:

$$
y\left(X_{t}\right)=\max _{j=1 \ldots C} \mu_{R_{j}}\left(X_{t}\right)
$$

The resulting fuzzy logic system then calculates the similarity of the input vector with the expected normal behavior training data.

\section{Neural Network based Signal Forecasting}

The dynamics of the sensory measurements are determined by the underlying physical (chemical) processes. For a complex system it is very difficult to model this dynamic behavior using conventional mathematical modeling. However, the dynamic temporal behavioral patterns can be modeled using machine learning techniques such as the feedforward Neural Network. In the third layer of the proposed data fusion system, the NN based signal forecasting block is used to forecast the near future behavior of the plant based on the intermediate previous historical data.

Fig. 3 depicts the implemented NN based signal forecasting block and the subsequent prediction error generation. The input vector to the feed-forward $\mathrm{NN}$ is constructed from the previous 3 sensory measurements as follows:

$$
X_{t}=\left\{\vec{x}_{t}, \vec{x}_{t-1}, \vec{x}_{t-2}, \vec{x}_{t}-\vec{x}_{t-1}, \vec{x}_{t-1}-\vec{x}_{t-2}\right\}
$$

A separate $\mathrm{NN}$ was trained for each forecasted attributed of the modeled system. The future $k$ values of the specific attribute are assigned as the desired forecasted output $\vec{y}_{t}$. The trained set of NNs predicts the future $k$ values of all monitored sensory measurements based on the available information.

Once the future $k$ samples of the actual plant behavior have been observed, they can be retrospectively matched against the original prediction and an error vector can be calculated. The $i^{\text {th }}$ attribute the prediction error $e_{t}^{i}$ for a plant signal forecasted at time $t$ can be calculated as follows:

$$
e_{t}^{i}=\frac{\sum_{j=1}^{k}\left|y_{t}^{j}-x_{t+j}^{i}\right|}{k}
$$

Hence, the NN based signal forecasting block generates a prediction error vector for all attributes of particular plant module. This error vector is transformed into a scalar robust anomaly indicator by a fuzzy logic controller as described in the following section.

\section{D.Fuzzy Logic based Alarm Generation}

One of the main goals of the implemented data-fusion system is to generate a small set of robust and easy-tounderstand alarms. The alarms are then presented to the operator through the HMI. For a specific plant module, the NN based signal forecasting block generates a vector of prediction errors for the set of available sensors. A Fuzzy Logic Controller (FLC) was implemented to fuse the prediction error into a robust scalar anomaly indicator [20].

The implemented FLC has two inputs. The first input is the maximum error $e_{\max }$ from the calculated prediction based error vector. The second input is the absolute value of the current gradient of the sensory input with the highest prediction error $g_{\max }$. After normalization, both inputs are fuzzified using two trapezoidal fuzzy sets Small and High as depicted in Fig. 4(a) and Fig. 4(b). The output of the anomaly indicator is modeled using three triangular fuzzy sets Small, Medium and High as depicted in Fig. 4(c), which express the anomaly level of the plant's behavior.

Table I show the used fuzzy rules and the respective output surface is depicted in Fig. 5. The used fuzzy rules suppress the amplitude of the produced anomaly indicator when a significant system transient is observed. The rationale behind this rule base is that it is reasonable to expect prediction error 

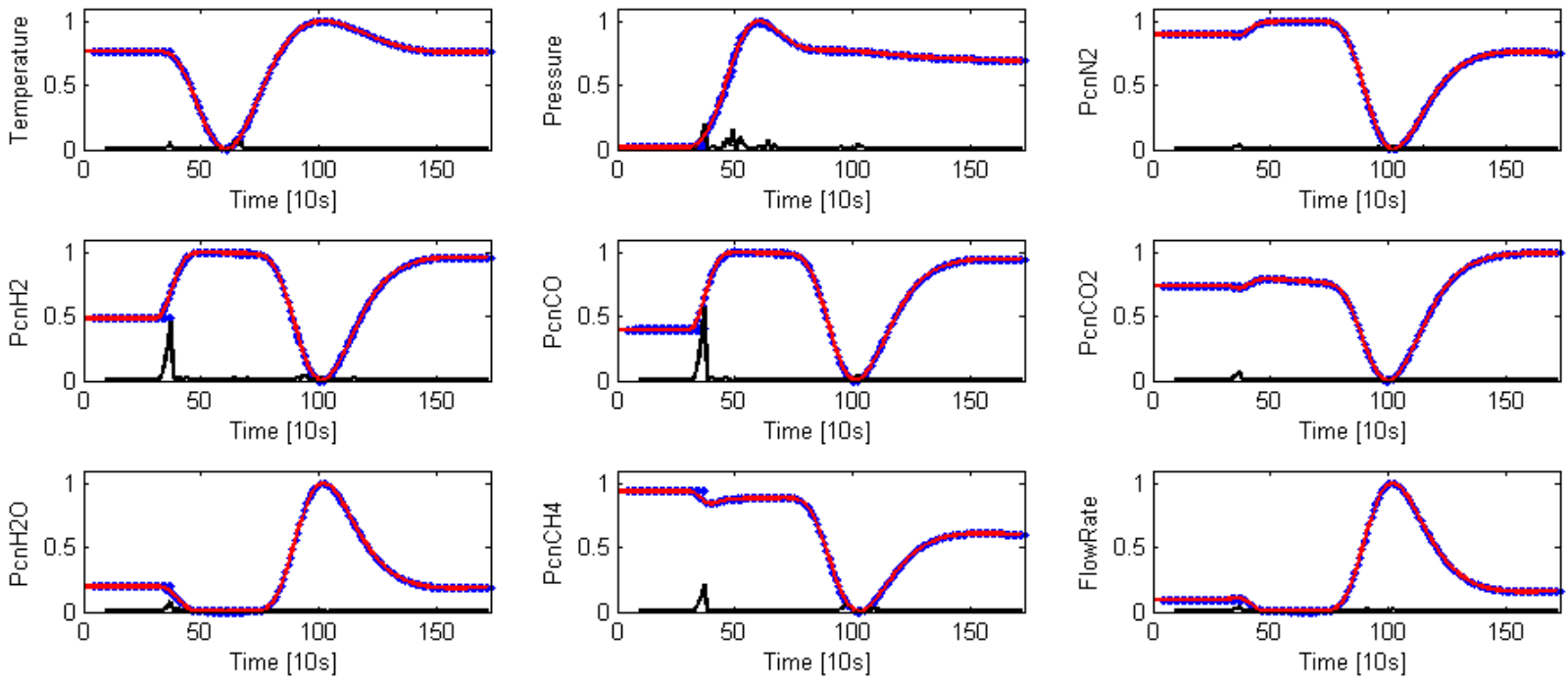

(a)

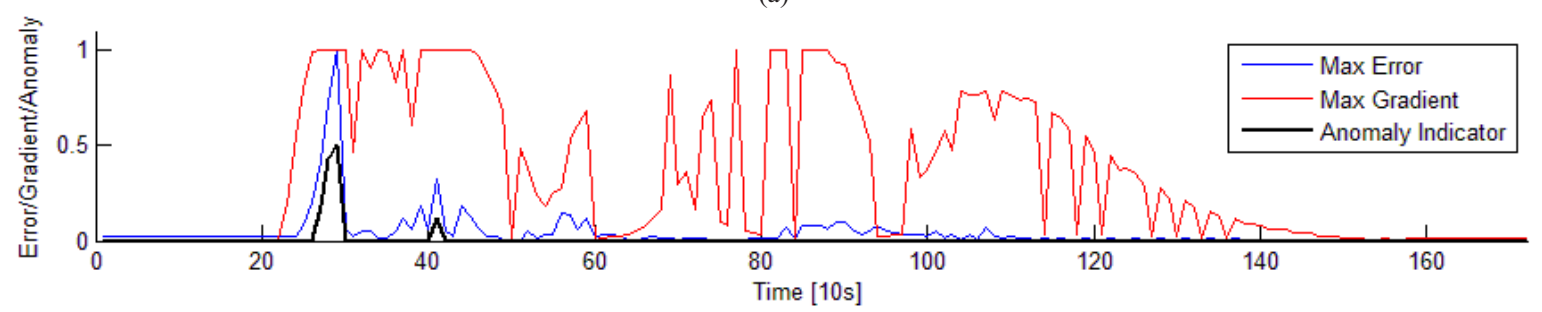

(b)

Fig. 6 Trended sensory measurements with the ANN signal prediction (a) and the fused anomaly indicator (b) for normal operation.

if the plant is in a dynamic transient behavior. However, a behavior prediction error generated during a steady behavior is likely an indicative of an anomaly.

\section{EXPERIMENTAL RESULTS}

This Section presents experimental testing of the implemented data fusion system. First, the considered experimental test-bed and the implemented test cases are described. Next, the performance of the data fusion system is demonstrated.

\section{A. Hytest Chemical Plan Model}

The used experimental test-bed was a Matlab Simulink model of the Idaho National Laboratory Hytest process, which is a testing facility for hybrid energy systems composed of tightly-coupled chemical processes [13], [14]. The system is composed of interconnected modules such as chemical reactors, heaters, condensers, storage tanks or compressors. Each Hytest module is equipped with a suite of 9 sensors measuring the stream flow rates, chemical compositions, pressure and temperature.

The Hytest Simulink model was integrated with the designed data fusion system as described in Section III. Future work will be focused on designing a novel HMI for increased state-awareness of the plant control using the anomaly indication from the proposed data fusion system.

\section{B. Test Cases}

The implemented Hytest model allows for generating both normal and abnormal operating conditions. For the purposes of performance evaluation of the implemented data fusion system the following test cases have been considered:
Test Case 1: Normal Steady Operations - The plant is in a stabilized steady state within the normal operating conditions.

Test Case 2: Normal Transient Operation - The plant is in a dynamic transient purposely introduced by the operator within the normal operating conditions.

Test Case 3: Component Failure in Transient Operation In this scenario, a specific component of the modeled plant is subject to physical failure (e.g. stuck valve), which prevents the plant transient to perform according to the normal behavior patterns.

Test Case 4: Intrusion Attempt in Steady Operation - Here, a component of the plant is compromised by an intrusion attack, which disrupts the steady state operation and forces the plant into a transient.

\section{C.Anomaly Detection}

Due to a limited space only the results of anomaly detection based of the prediction errors are demonstrated for test cases 2, 3 and 4. In the testing scenarios, the sensors have been periodically sampled with a period of 10 seconds. The NNbased forecaster was trained on the three most recent sensory readings as described in (10). For a single module with a suite of 9 sensors this resulted in 45-dimensional input vector for the NN. A dedicated NN was trained for prediction of the intermediate 6 future measurements for each sensors $\backslash$, thus forecasting the next 1 minute of plant's behavior.

Fig. 6(a) shows the trended sensory variables during normal Hytest transient as recorded from one of the Hytest valves. The red line shows the true observed value. The overlapping blue dots show the next predicted 1 minute of plant behavior for each time sample. Finally, the black line denotes the prediction error. It can be observed that the beginning of the 

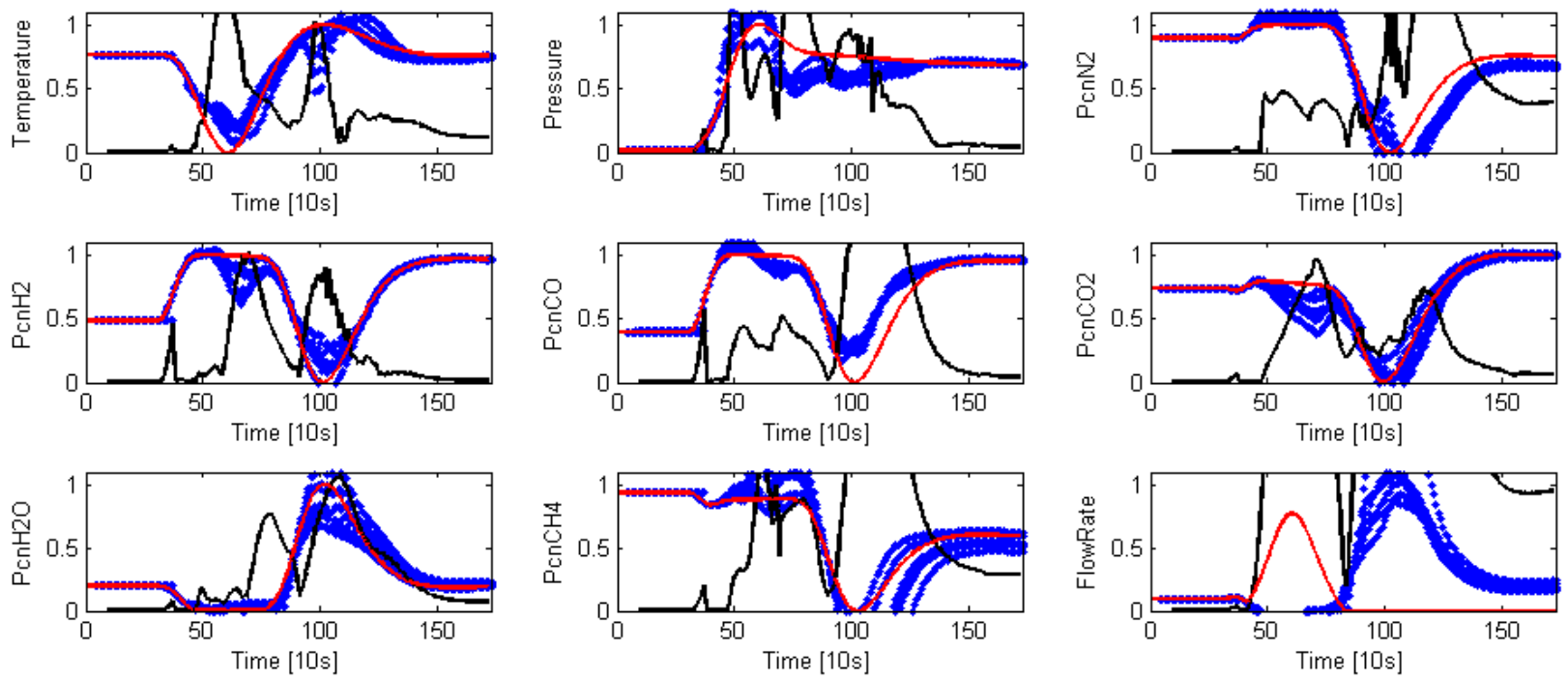

(a)

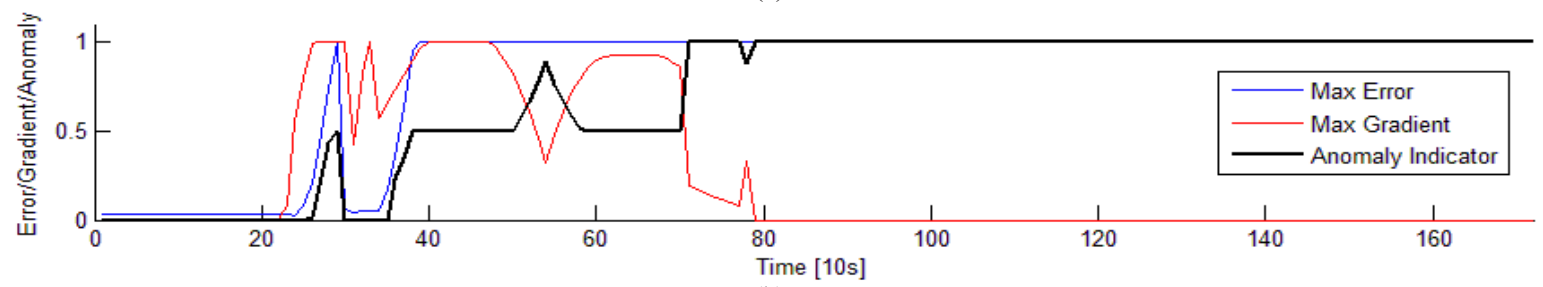

(b)

Fig. 7 Trended sensory measurements with the ANN signal prediction (a) and the fused anomaly indicator (b) for stuck valve.
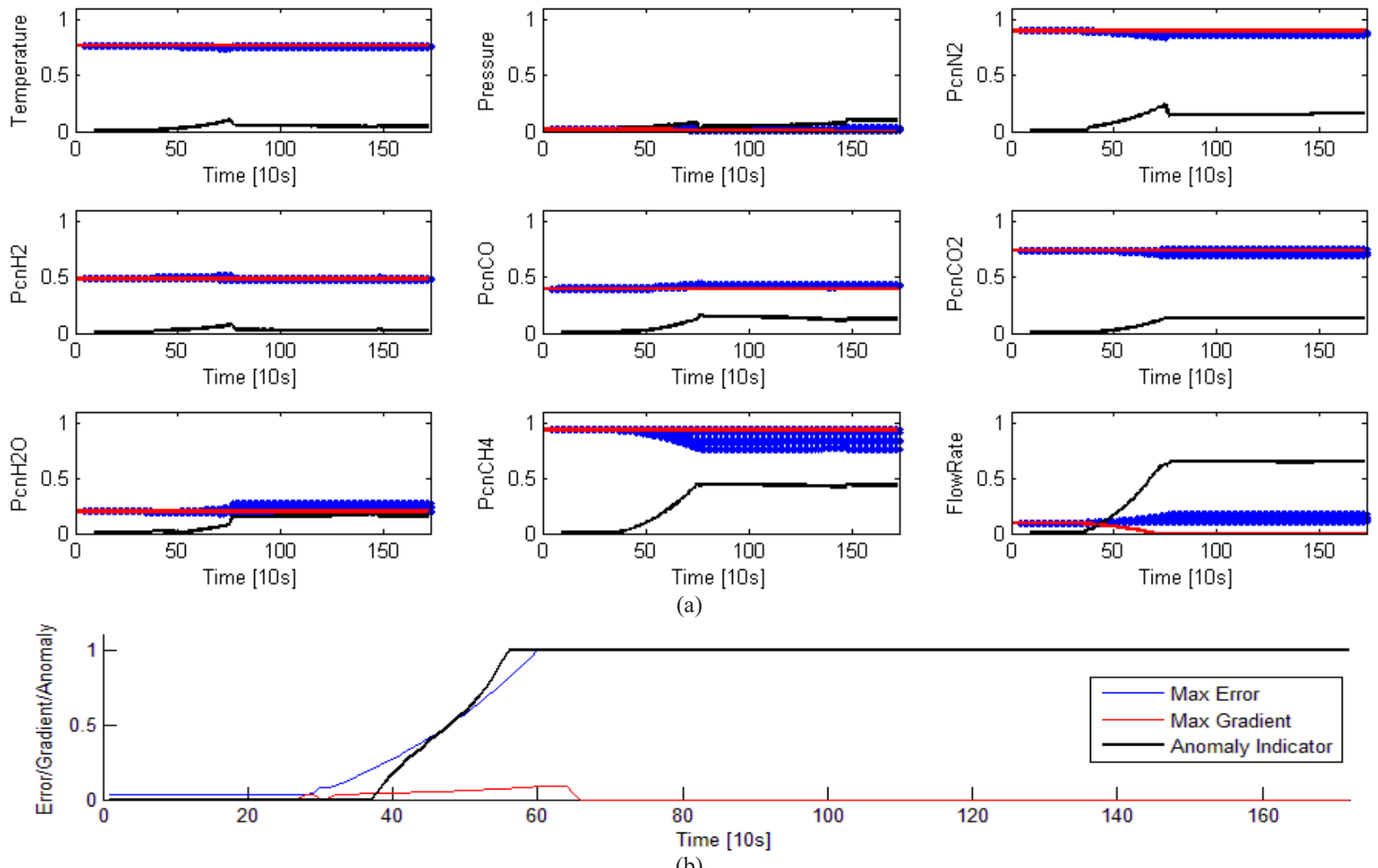

(b)

Fig. 8 Trended sensory measurements with the ANN signal prediction (a) and the fused anomaly indicator (b) for an intrusion.

plant transient at time 300 seconds triggered prediction error due to unexpected gradient. Fig. 6(b) then shows the fused anomaly indicator. It can be seen that the FLC suppresses the amplitude of the anomaly indicator for the beginning of the transient due to high gradient of the sensory inputs.
Fig. 7(a) shows the prediction error for the case of stuck valve. Because the stuck valve substantially changes the behavior of the plant model, the predicted signals do not match the observed behavior and high prediction errors are 
reported. Fig. 7(b) shows that the increased prediction error resulting in a timely reporting of this anomalous behavior.

Finally, Fig. 8(a) shows the prediction errors due to malicious attack, when an intruder took control of the valve's controller and started to gradually open this valve. Again, this unforeseen control action resulted in a mismatch between the predicted and the observed behavior. This is then reflected in the increased amplitude of the anomaly indicator as shown in Fig. 8(b).

\section{V.CONCLUSION}

This paper presented the design of a fuzzy-neural based data fusion system for increased state-awareness of resilient control systems. The implemented data fusion system consists of conventional threshold based alarms, anomalous behavior detector using self-organizing maps, and prediction error based alarms using neural network signal forecasting. The proposed system was integrated with a model of the Idaho National Laboratory Hytest hybrid energy systems testing facility.

The presented experimental results demonstrated the benefits of the developed data fusion system in terms of improved cyber-security and state-awareness of the critical control system. The calculated anomaly indicators were shown to correctly predict the future plant's normal behavior and detect anomalous behavior due to both system component failure and malicious network attack. Future work is intended to investigate the possibilities of further classifying the causes of observed anomalous behavior and integrating the results of the data-fusion system into an HMI. Furthermore, the performance validation, parameters tuning and resiliency improvement assessment of the designed data fusion engine is currently an ongoing research effort.

\section{ACKNOWLEDGMENT}

Work supported by the U.S. Department of Energy under DOE Idaho Operations Office Contract DE-AC07-05ID14517, performed as part of the Instrumentation, Control, and Intelligent Systems Distinctive Signature (ICIS) of Idaho National Laboratory.

\section{REFERENCES}

[1] C. G. Rieger, D. I. Gertman, M. A. McQueen, "Resilient Control Systems: Next Generation Design Research," in Proc. $2^{\text {nd }}$ IEEE Conf. on Human System Interactions, Catania, Italy, pp. 632-636, May 2009.

[2] S. S. Choi, K. S. Kang, H. G. Kim, S. H. Chang, "Development of an On-Line Fuzzy Expert System for Integrated Alarm Processing in Nuclear Power Plants," in IEEE Trans. on Nuclear Science, vol. 42, issue: 4, pp. 1406-1418, Aug. 1995.
[3] J. Rabatel, S. Bringay, P. Poncelet, "Fuzzy Anomaly Detection in Monitoring Sensor Data," in Proc. World Congress on Computational Intelligence, Barcelona, Spain, July 2010.

[4] K. Hadad, M. Pourahmadi, H. Majidi-Maraghi, "Fault diagnosis and classification based on wavelet transform and neural network," in Progress in Nuclear Energy, vol. 53, issue: 1, pp. 41-47, Jan. 2011.

[5] H. Shen, J. M. Doster, "Application of a neural network based feedwater controller to helical steam generators," in Nuclear Engineering and Design, vol. 239, issue: 6, pp. 1056-1065, June 2009.

[6] M. Fast, T. Palme. "Application of artificial neural networks to the condition monitoring and diagnosis of a combined heat and power plant," in Energy, vol. 35, issue: 2, pp. 1114-1120, February2010.

[7] K. Nabeshima, T. Suzudo, T. Ohno, K. Kudo, "Nuclear reactor monitoring with the combination of neural network and expert system," in Mathematics and Computers in Simulation, vol. 60, issue: 3-5, pp. 233-244, Sep. 2002.

[8] R. Razavi-Far, H. Davilu, V. Palade, C. Lucas. "Model-based fault detection and isolation of a steam generator using neuro-fuzzy networks," in Neurocomputing, vol. 72, issues: 13-15, pp. 2939-2951, August 2009.

[9] S.-L. Hwang, J.-T. Lin, G.-F. Liang, Y.-J. Yau, T.-Ch. Yenn, Ch.-Ch. Hsu, "Application control chart concepts of designing a pre-alarm system in the nuclear power plant control room," in Nuclear Engineering and Design, vol. 238, issue: 12, pp. 3522-3527, Dec. 2008.

[10] K. Salashoor, M Kordestani, M. S. Khoshro, "Fault detection and diagnosis of an industrial steam turbine using fusion of SVM (support vector machine) and ANFIS (adaptive neuro-fuzzy inference system) classifiers," in Energy, vol. 35, issue: 12, pp. 5472-5482, December 2010.

[11] O. Linda, T. Vollmer, J. Wright, M. Manic, "Fuzzy Logic Based Anomaly Detection for Embedded Network Security Cyber Sensor," in Proc. IEEE Symposium Series on Computational Intelligence, Paris, France, April, 2011.

[12] O. Linda, T. Vollmer, M. Manic,"Neural Network Based Intrusion Detection System for Critical Infrastructures, " in Proc. Int. Joint INNSIEEE Conf. on Neural Networks, Atlanta, Georgia, June 14-19, 2009.

[13] C. Stoots, L. Shun, J. O'Brien, "Integrated Operation of the INL Hytest System and High-Temperature Steam Electrolysis for Synthetic Natural Gas Production," in Proc. of $2^{\text {nd }}$ International Meeting of the Safety and Technology of Nuclear Hydrogen Production, Control and Management, June 2010.

[14] L. Shun, R. Boardman, S. Cherry, C. Rieger, "HYTEST Phase I Facility Commissioning and Modeling," INL External Technical Report, INLEXT-09-16961, Sep. 2009.

[15] P. J. Werbos, The Roots of Backpropagation, New York: Johns Wiley \& Sons, 1994.

[16] D. Marquardt, "An algorithm for least squares estimation of non-linear parameters," in J. Soc. Ind. Appl. Math., pp.431-441, 1963.

[17] M. Hagan, M. Menhaj, "Training feedforward networks with the Marquardt algorithm," in IEEE Transaction on Neural Networks, vol. 5, no. 6, pp. 989-993, 1994.

[18] T. Kohonen, "Automatic Formation of Topological Maps of Patterns in a Self-Organizing System, " in Proc. SCIA, E. Oja, O. Simula, Eds. Helsinki, Finland, pp. 214-220, 1981.

[19] S. Haykin, Neural Networks and Learning Machines - Third Edition, Prentice Hall, 2008.

[20] J. M. Mendel, Uncertain Rule-Based Fuzzy Logic Systems: Introduction and New Directions, Prentice-Hall, Upper Saddle River, NJ, 2001. 